\title{
Intraspecific evolution of Canary Island Plecotine bats, based on mtDNA sequences
}

\author{
$\mathrm{J} \mathrm{Pestano}^{1}$, RP Brown ${ }^{2}$, NM Suárez ${ }^{1}, \mathrm{~J} \mathrm{Benzal}^{3}$ and $S$ Fajardo ${ }^{4}$ \\ ${ }^{1}$ Departamento de Genética, Facultad de Medicina, Universidad de Las Palmas de Gran Canaria, 35080 Las Palmas, Spain; ${ }^{2}$ School of \\ Biological and Earth Sciences, Liverpool John Moores University, Byrom St., Liverpool L3 3AF, UK; ${ }^{3}$ Estación Experimental de \\ ZonasÁridas (CSIC), C/ General Segura, 104001 Almería, Spain; ${ }^{4}$ Viceconsejería de Medio Ambiente, Gobierno de Canarias, 38071 La \\ Laguna, Tenerife, Spain
}

\begin{abstract}
Island differentiation and relationships with congenerics were investigated in the endemic Canary Island bat Plecotus teneriffae, based on $\sim 1 \mathrm{~kb}$ of mtDNA from the $16 \mathrm{~S}$ rRNA and cytochrome $b$ genes. $P$. teneriffae had closer affinities with $P$. austriacus than with $P$. auritus. Levels of differentiation between Canary Islands were quite high relative to Pipistrelle-like bats, consistent with philopatric behaviour in the Plecotus genus. Cladogenesis within $P$. teneriffae appears to have occurred after the emergence of the islands of El Hierro and La Palma during the Pleistocene. An intraspecific
\end{abstract}

network shows that haplotypes from the younger islands of La Palma and El Hierro are connected to the Tenerife haplotype by a similarly large number of mutational steps. This suggests that they were both colonised at a similar time from the much older island of Tenerife. The other Plecotine bat species, Barbastellus barbastellus shows close affinities with $B$. barbastellus from mainland Spain, with levels of mtDNA divergence being comparable with intraspecific variation within other mammal species.

Heredity (2003) 90, 302-307. doi:10.1038/sj.hdy.6800240

Keywords: chiroptera; cytochrome $b$; ecology; phylogeny; phylogeography; 16S rRNA

\section{Introduction}

The Canary archipelago has become a model region for island phylogeographical research with a series of studies that have focused on lizards, insects, spiders and plants (reviewed in Juan et al, 2000). Bats provide an interesting additional group: they have very different biologies and in particular very different dispersal capabilities, compared with many of the aforementioned taxa. For example, it is of biogeographical interest to know whether they show patterns of mtDNA differentiation between islands that reflect relatively recent colonisation of new islands, as seen in nonvolant vertebrates such as lizards (González et al, 1996; Brown and Pestano, 1998). Studies of genetic differentiation will also throw new light on the ecology and behaviour of this group (Burland and Wilmer, 2001).

The Canary Island archipelago consists of seven main islands and some small associated islets. Bats are one of only two groups of extant endemic mammals (one of the Canary Island shrews also appears to be an endemic), and therefore demand high conservation priority. Seven species of bat are known (Trujillo, 1991) although the only currently recognised endemic is the Tenerife long-eared bat Plecotus teneriffae. This has been recorded on the Western islands of Tenerife, La Palma and El Hierro, with colonies found mainly in caves and

Correspondence: RP Brown, School of Biological and Earth Sciences, Liverpool John Moores University, Byrom St., Liverpool L3 3AF, UK. E-mail: r.p.brown@livjm.ac.uk

Received 4 March 2002; accepted 6 December 2002 volcanic lava tubes. It may also be present on the island of La Gomera (situated between Tenerife and El Hierro) although there are no definite records (Trujillo, 1991). The other Plecotine species, Barbastellus barbastellus, has been reported from only two islands, Tenerife and La Gomera.

Over the last century there has been considerable discussion over the species status of $P$. teneriffae. It was originally included with the widespread European/ Asian brown long-eared bat, P. auritus, but subsequently included with P. austriacus (the grey long-eared bat) from S. Europe, Asia, N. Africa and W. Africa) when this taxon was split from the former (reviewed by Trujillo, 1991; Benzal and Fajardo, 1997). The authors of an alpha taxonomy study considered that it was worthy of full species status after showing that it was morphologically differentiated from both $P$. auritus and $P$. austriacus (Ibáñez and Fernández, 1985).

Canary B. barbastellus have also been compared with $B$. barbastellus from mainland Spain (Trujillo, 1991). External and cranial morphology was reported to be similar, but some pelage colour differences were noted with the author suggesting that species or subspecies recognition may be justified.

This mtDNA analysis of the Plecotine tribe is part of a larger revision of the Canary Island bats that has already uncovered low but significant between island mtDNA diversity in other Vespertilionid species (Pestano et al, 2003). It aims to evaluate whether any species appear to have been introduced from mainland Spain during recent times; but more importantly establish levels of biodiversity between islands Canary Island to underpin local conservation strategies. 


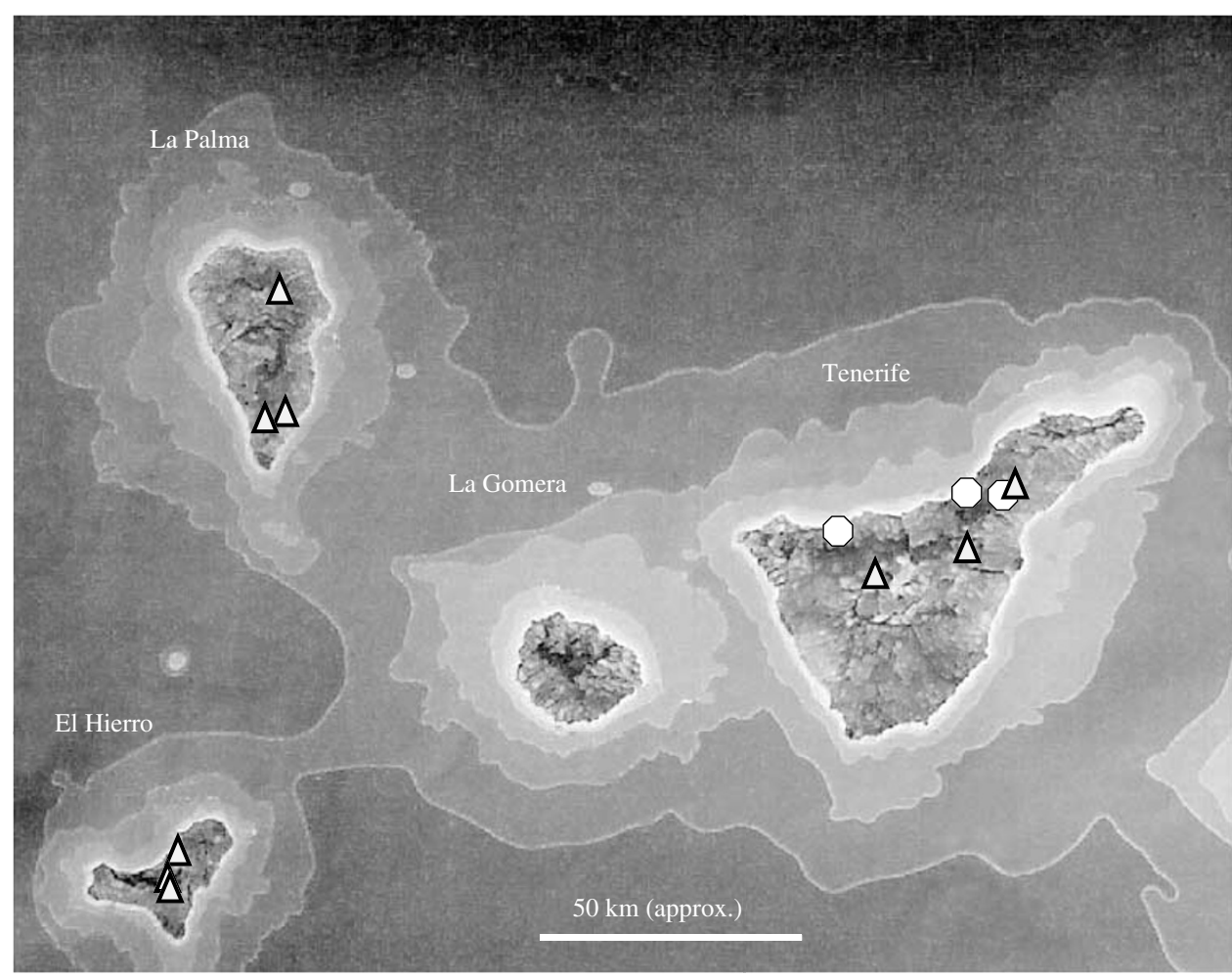

Figure 1 Capture sites for P. teneriffae $(\triangle)$ and B. barbastellus $(O)$ in the Canary Islands. See Table 1 for locality names and numbers of individuals.

\section{Materials and methods}

During the aforementioned project on Canary Island microchiroptera, suitable refuges were investigated on all islands between April 1999 and September 2001. Bat detectors were used to detect the presence of Barbastellus. Plecotus were captured using either mist nets placed on the entrance to refuges, or by hand in the refuges during the day. This strategy was used on all the western islands but Plecotus were only seen/caught on El Hierro, La Palma and Tenerife, in accordance with its known distribution (Table 1, Figure 1). Barbastellus was only detected on Tenerife, despite having previously been recorded from La Gomera (Trujillo, 1991). Blood samples were taken from a wing vein and the individuals released. We also captured and blood-sampled three P. auritus from Valsaín, Segovia (N. Spain), six P. austriacus from Viana de Cega, Monte Arenas and Pedrajas in Valladolid (also N. Spain), and a B. barbastellus from Peñas Meleras, Zaragoza (N. Spain). The blood was dried on filter paper and DNA extracted using a MachereyNagel Nucleospin ${ }^{\mathbb{R}} \mathrm{C}+\mathrm{T}$ kit. Fragments of the cytochrome $b$ gene were amplified using the following light strand (forward) primer for P. teneriffae, P. auritus and all B. barbastellus: L14841 5'-CCATCCAACATCTCAGCATGATGAAA-3' (Kocher et al, 1989). The corresponding primer for $P$. austriacus was 5'-TGACCAACATTCGAAAATCCC-3'. Heavy strand primers were: H15485 5'-TTGCTGGGGTGAAGTTTTCTGGGTC-3' for $P$. teneriffae and $P$. auritus, 5'-TTGCTGGGGTGAAGTTTTCTGG-3' for all B. barbastellus and H15915 5'GTCTTCAGTTTTTGGTTTACAAGAC- $3^{\prime}$ for $P$. austriacus (Fu et al, 2000). The same (labelled) primers used in the
PCRs were also used for sequencing although the following additional primers were used to clarify certain regions: (1) light strand, $P$. austriacus and Canary $B$. barbastellus 5'-TGAGGACAAATATCCTTCTGAGG-3', (2) heavy strand, Canary B. barbastellus 5'-GGATTAGATCCTGTCTCGTG-3', (3) heavy strand, P. austriacus 5'GCCCCTCAGAATGATATTTGTCCTCA- ${ }^{\prime}$. A partial $16 \mathrm{~S}$ rRNA gene sequence was amplified and sequenced using the primers: $5^{\prime}$-CGCCTGTTTAACAAAAACAT- $3^{\prime}$ (light strand) and 5'-CCGGTCTGAACTCAGATCACGT-3' (heavy strand) (Simon et al, 1990). A Pharmacia ALF-express sequencer was used for sequencing. Homologous sequences were available from two other Vespertilionid bats from mainland Spain: Pipistrellus kuhli and Hypsugo savii (see EMBL accession numbers AJ426619, AJ426640, AJ426620, AJ426641).

ClustalX was used to align sequences (Thompson et al, 1997). The initial 16S rRNA alignment was subsequently compared with the secondary structure model proposed for $P$. townsendii to assess whether indels were correctly positioned (Van de Peer et al, 2000). The congruence of the signal provided by the two genes was investigated using the incongruence length difference test (ILD) (Farris et al, 1994). The 16S rRNA and cytochrome $b$ sequences were subsequently joined and haplotypes identified for subsequent analyses.

Phylogenetic relations between the three main taxa were explored using maximum likelihood (ML) and unweighted parsimony (MP). MODELTEST (ver. 3.06) (Posada and Crandall, 1998) and PAUP* (ver. 4.0 b8) (Swofford, 1998) were used to obtain the ML scores for 56 models of DNA substitution for a simple neighbour joining tree (based on Jukes Cantor distances, outgroup 
Table 1 Specimens, localities and haplotypes for the Canary Island bats used in the study

\begin{tabular}{|c|c|c|}
\hline Species/island & Capture site & Haplotype \\
\hline P. teneriffae Tenerife & Cueva de Cosme-Caldera de Pedro Gil $(n=3)$ & Pt TF \\
\hline P. teneriffae Tenerife & Galeria los Berros-La Esperanza $(n=1)$ & $\mathrm{Pt}$ TF \\
\hline P. teneriffae Tenerife & Cueva Tiro del Guanche-Parque Nacional del Teide $(n=1)$ & $\mathrm{Pt} \mathrm{TF}$ \\
\hline P. teneriffae La Palma & Salto del Tigalate $(n=1)$ & Pt LP\#01 \\
\hline P. teneriffae La Palma & Marcos y Corderos $(n=2)$ & Pt LP\#02 \\
\hline P. teneriffae La Palma & Llano los Cestos $(n=1)$ & Pt LP\#01 \\
\hline P. teneriffae El Hierro & La Majada $(n=2)$ & Pt EH\#01 \\
\hline P. teneriffae El Hierro & Jinama $(n=1)$ & Pt EH\#02 \\
\hline P. teneriffae El Hierro & La Cueva de Mocan $(n=1)$ & Pt EH\#02 \\
\hline B. barbastellus Tenerife & Los Berros-La Esperanza $(n=3)$ & $\begin{array}{l}\text { Bb TF\#01 (1) } \\
\text { Bb TF\#02 (2) }\end{array}$ \\
\hline B. barbastellus Tenerife & La Labrada-El Sauzal $(n=2)$ & $\mathrm{Bb}$ TF\#01 \\
\hline B. barbastellus Tenerife & $\operatorname{Icod}(n=2)$ & $\begin{array}{l}\text { Bb TF\#01 } \\
\text { Bb TF\#02 }\end{array}$ \\
\hline
\end{tabular}

Numbers in parentheses after haplotypes are used where more than one haplotype was obtained at a capture site

Table 2 Matrix of uncorrected distances (number of differences) between islands/taxa for cytochrome $b$ (lower diagonal) and 16S rRNA (upper diagonal)

\begin{tabular}{|c|c|c|c|c|c|c|c|c|c|c|}
\hline & Pt LP\#01 & Pt LP\#02 & Pt TF & Pt EH\#01 & Pt EH\#02 & P. austriacus & P. auritus & Bb TF01 & Bb TF02 & Bb Zaragoza \\
\hline Pt LP\#01 & & 0 & 2 & 5 & 5 & 25 & 38 & 68 & 67 & 66 \\
\hline Pt LP\#02 & 3 & & 2 & 5 & 5 & 25 & 38 & 68 & 67 & 66 \\
\hline Pt TF & 6 & 9 & & 3 & 3 & 25 & 40 & 69 & 68 & 67 \\
\hline Pt EH\#01 & 7 & 10 & 5 & & 0 & 26 & 41 & 66 & 65 & 64 \\
\hline Pt EH\#02 & 8 & 11 & 6 & 1 & & 26 & 41 & 66 & 65 & 64 \\
\hline$P$. austriacus & 58 & 59 & 60 & 57 & 58 & & 41 & 71 & 70 & 69 \\
\hline$P$. auritus & 73 & 75 & 77 & 76 & 77 & 75 & & 68 & 67 & 69 \\
\hline Bb TF01 & 92 & 94 & 93 & 94 & 95 & 94 & 102 & & 1 & 11 \\
\hline Bb TF02 & 92 & 94 & 93 & 94 & 95 & 94 & 102 & 0 & & 12 \\
\hline Bb Zaragoza & 96 & 97 & 95 & 96 & 97 & 100 & 102 & 21 & 21 & \\
\hline
\end{tabular}

taxa not used). This was carried out on the entire sequence as differences in DNA evolution between genes are merely an extension of evolution within genes, and this heterogeneity can be incorporated within a sufficiently complex model. Hierarchical testing of models was used to assess the model with the best fit. The ML tree was then computed (heuristic search with random, replicated addition of taxa), and bootstrapping (500 replicates) used to assess support for internal nodes. Intraspecific differentiation within $P$. teneriffae was assessed using intraspecific unrooted 'cladograms' based on a statistical parsimony procedure that has been shown to have greater statistical power and accuracy when there are few variable sites (Templeton et al, 1992; Crandall, 1994). Sequences were connected under the $95 \%$ probability of parsimony criterion using the software TCS (Clement et al, 2000).

\section{Results}

A total of 511 and 508-512 homologous base pairs of the cytochrome $b$ and 16S rRNA sequences, respectively, were obtained in all specimens (EMBL accession numbers: AJ431644-AJ431663). The length of the 16S rRNA fragment varied because of indels, but none appeared to be involved in secondary structure interactions (one was located in a stem area, but corresponded to an internal bulge). There were 153 variable sites (110 parsimony informative) in the cytochrome $b$ fragment with 96 (74 parsimony informative) in the $16 \mathrm{~S}$ rRNA, excluding outgroup taxa. Uncorrected distances between taxa are shown in Table 2 for each gene individually. Overall mean uncorrected sequence divergence was $0.8-1.6 \%$ between islands for P. teneriffae, $11.2 \% / 8.2 \%$ between $P$. teneriffae and $P$. auritus/P. austriacus, respectively, and $3.2 \%$ between $B$. barbastellus from Tenerife and mainland Spain and $15.3 \%$ between Barbastellus and Plecotus.

There was no evidence that the two partial genes provided trees that were significantly shorter (when summed) than the overall tree. In fact, the sum of lengths (539) of the MP trees for each individual fragment was identical to the length of the most parsimonious tree for all data that gave $P=1.0$ (500 replicates) for the ILD test.

The best model of DNA substitution was the TamuraNei model (Tamura and Nei, 1993) with $\gamma$ distributed site heterogeneity. Parameter estimates were: base frequencies 0.3171 (A), 0.2449 (C), 0.1661 (G), 0.2719 (T); rate estimates 7.9983 for A-G, 14.4965 for C-T and unity for all other substitutions; gamma distribution with shape parameter $\alpha=0.2459$. The ML tree found under this model had a log-likelihood score of -3676.72 (using eight substitution categories to represent the between-site rate heterogeneity). Bootstrap support was quite high for most internal nodes, although monophyly of Tenerife/La Palma haplotypes was not strongly supported (Figure 2). There was no significant difference between the log- 


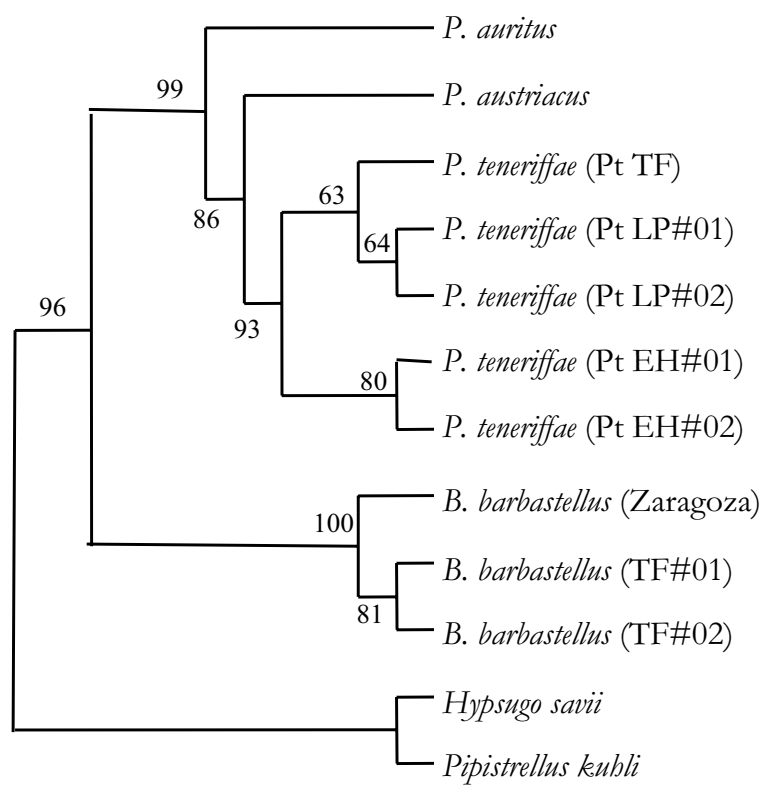

Figure 2 ML tree obtained for all Canary taxa based on cytochrome $b$ and 16S rRNA sequences. Internal labels are bootstrap support values.

likelihood scores of this ML tree and the same tree computed under a molecular clock assumption $\left(2 \delta=12.13, P=0.27\right.$ (based on $\chi^{2}$ with $\left.10 \mathrm{df}\right)$ ).

The MP tree gave the same interspecific patterns, but there were differences in the hypothesized relations within P. teneriffae: the Tenerife populations appeared monophyletic with El Hierro relative to La Palma, rather than vice versa (not shown). However, four parsimony trees were notably shorter than the rest with either 539 (one tree, as described) or 540 (three trees) steps. One of these also supported the (El Hierro, (La Palma, Tenerife)) relation found using the ML approach suggesting that there is not such a conflict between the two methods as might first appear.

The intraspecific cladogram (Figure 3) reveals more information on the relationships between these taxa by showing that both La Palma and El Hierro haplotypes are separated from Tenerife by a similar (and considerable) number of mutational steps. This similarity may help explain the aforementioned ambiguity over the relationships within $P$. teneriffae. It is also interesting to note that the two different haplotypes within La Palma correspond to the different northern and southern samples of $P$. teneriffae, suggesting possible within-island divergence.

\section{Discussion}

The relationships within $P$. teneriffae are difficult to resolve, but given the intraspecific cladogram the reason is apparent: a similar number of mutational steps separate La Palma and El Hierro from the ancestral haplotype. This suggests a 'hard' polytomy arising from cladogenesis at similar points in time, possibly because of the 'simultaneous' (in geological time) colonisation of these latter two islands.

Although the phylogeny tells us about the pattern of historical cladogenesis, it does not allow definitive

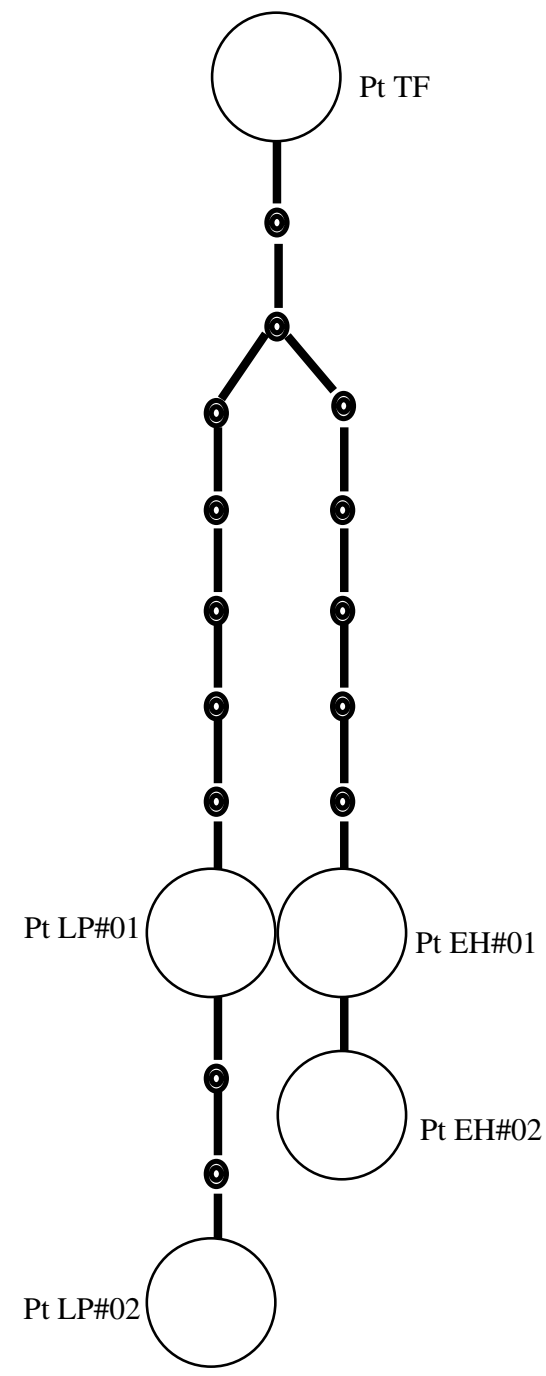

Figure 3 Parsimony network connecting the five $P$. teneriffae haplotypes found on Tenerife, La Palma and El Hierro. Missing haplotypes are designated by the symbol $\odot$

inferences about colonisation because the geographical location corresponding to each ancestral node is unknown. This problem can sometimes be overcome using geological data (Brown and Pestano, 1998). The oldest parts of Tenerife were formed during the Miocene, approximately 11.6 Ma (Ancochea et al, 1990), making it much older than either El Hierro ( 1.2 Ma, (Guillou et al, 1996)) or La Palma (oldest subaerial components date to $\sim 1.7 \mathrm{Ma}$, (Guillou et al, 2001)). Sequence divergence is low between $P$. teneriffae from the latter two islands and Tenerife (means of 1.1 and $1.5 \%$ for cytochrome $b$ and 0.6 and $0.4 \%$ for $16 \mathrm{~S}$ rRNA for El Hierro and La Palma, respectively). Tenerife-El Hierro divergence is lower than divergence between the adjacent islands of $\mathrm{La}$ Gomera and El Hierro for the bat Hypsugo savii and giant Gallotia lizards (Pestano et al, 2003), and Chalcides skinks (Brown and Pestano, 1998), although higher than between other islands for Hypusgo and Pipistrellus (see later). Bats might be expected to be better island colonisers than lizards and so it is possible that the lower divergence in Plecotus is because of a later arrival in the archipelago as a whole. 
Under published evolutionary rates for the two genes (see Mindell and Thacker, 1996; Pesole et al, 1999; Avise, 2000) colonisation appears to have occurred quite a long time after appearance of the newer islands, so the possibility that one or both of these events represents a back-colonisation (ie, new to old island) cannot be completely ruled out. However, the longer branch lengths associated with the newer islands suggest that this is not the case: divergence might be accelerated in a founder population because of small effective size. This is consistent with colonisation of El Hierro and La Palma from Tenerife as there are a much greater number of mutational steps between the former and the internal node in the network. This scenario would be more robust if data were available from La Gomera, the island adjacent to Tenerife. While we do not rule out the possibility that this species exists there, there appears to be fewer suitable Plecotus refuges relative to the other western islands (see Trujillo, 1991). Owing to its intermediate position, this island was likely to have been involved in the historical range expansion from Tenerife to El Hierro, but whether this involved full island colonisation or not is unknown.

The degree of differentiation between islands is generally higher than that previously described between Pipistrellus maderensis from Tenerife, La Palma, La Gomera and El Hierro based on homologous mtDNA sequences (Pestano et al, 2003). It is also greater than that between $H$. savii from La Gomera, Tenerife and Gran Canaria, although slightly less than the differentiation between these populations and the more distant El Hierro population (as mentioned previously). Owing to the small sample sizes, we cannot completely rule out the possibility that maternal lineages could be shared between islands. Note that this has been observed for the bat Artibeus jamaicensis in the Caribbean (Pumo et al, 1988). However, if we assume that this is not the case (and so haplotype differences are representative of general differences between the islands), then it would seem likely that dispersal is low in Plecotus. This is not surprising given that the morphology of Plecotus bats does not favour fast, long distance flight (Norberg, 1976). Furthermore, they show high-site fidelity to volcanic lava tubes and caves and low natal dispersal (Fajardo, unpublished data).

A recent study on the congeneric species $P$. auritus would support the idea of low dispersal leading to considerable differentiation (Burland et al, 1999). This is a highly philopatric species and gene flow appears to be determined primarily by extra-colony copulation rather than natal dispersal. This gives rise to detectable genetic differentiation among colonies within a single river valley. Similar high female site fidelity has also been forwarded as an explanation of mtDNA differentiation between sites in the ghost bat Macroderma gigas from $\mathrm{N}$. Australia (Worthington-Wilmer et al, 1994).

The closest taxon to $P$. teneriffae is $P$. austriacus, rather than $P$. auritus. Although this appears to resolve an earlier dispute (Ibáñez and Fernández, 1985), it does not necessarily identify the sister taxon to P. teneriffae. As yet unpublished studies suggest that $P$. teneriffae is most closely related to the North African P. christei (Ibañez and Juste, personal communication).

$B$. barbastellus is divergent relative to the $B$. barbastellus haplotype from mainland Spain, but possibly less than might be expected between these two isolated populations. In fact, the divergence between geographically closer Moroccan and Spanish populations of Myotis myotis is much higher (approximately $10 \%$ for cytochrome b) (Castella et al, 2000) than that observed between Spanish and Canary Island populations of $B$. barbastellus $(4.0 \%$ for the same gene). It has been proposed that the former be treated as separate species, however, it would appear premature to make a similar proposal for B. barbastellus.

In sum, the Tenerife long-eared bat, $P$. teneriffae shows interesting phylogeographical patterns between the three Canary Islands of Tenerife, La Gomera and El Hierro, which appear to be because of relatively low levels of between-island dispersal. This differentiation needs to be taken into account for conservation purposes because it clearly contributes to the genetic diversity of this species. Furthermore, the apparently low levels of betweenisland dispersal mean that the probability of recolonisation of an island (after extinction) from a neighbour would be negligible.

\section{Acknowledgements}

Richard Brown undertook the sequence analyses and preparation of the manuscript during an EC Marie Curie senior research fellowship (Category 40: HPMF-CT-200000886). We thank the Viceconsejería de Medio Ambiente, Tenerife for motivating, financing and granting permission for the current study. Many people contributed to this project. These include Manuel Santana, Juan Carlos Batista, Felix Medina, Guillermo Delgado and Nelly Furtado. Cartografica de Canarias, SA (Grafcan) granted permission for modification/publication of Figure 1, which was based on an orthophoto of the Canary Islands (http://www.grafcan.com). We thank two anonymous reviewers for comments on an earlier version of the manuscript.

\section{References}

Ancochea E, Fuster JM, Ibarrola E, Cendrero A, Coello J, Hernan F, Cantagrel JM, Jamond C (1990). Volcanic evolution of the island of Tenerife (Canary Islands) in the light of new K-Ar data. J Volcanol Geothermal Res 44: 231-249.

Avise JA (2000). Phylogeography: the History and Formation of Species. Harvard University Press: Cambridge, MA.

Benzal J, Fajardo S (1997). Murciélago Orejudo Canario Plecotus teneriffae (Barret-Hamilton, 1907). Galemys 9: 4-14.

Burland TM, Barratt EM, Beaumont MA, Racey PA (1999). Population genetic structure and gene flow in a gleaning bat, Plecotus auritus. Proc R Soc Lond B 266: 975-980.

Burland TM, Wilmer JW (2001). Seeing in the dark: molecular approaches to the study of bat populations. Biol Rev 76: 389-409.

Brown RP, Pestano J (1998). Phylogeography of skinks (Chalcides) in the Canary Islands inferred from mitochondrial DNA sequences. Mol Ecol 7: 1183-1191.

Castella V, Ruedi M, Excoffier L, Ibañez C, Arlettaz R, Hausser J (2000). Is the Gibraltar Strait a barrier to gene flow for the bat Myotis myotis (Chiroptera: Vespertilionidae)? Mol Ecol 9: 1761-1772

Clement M, Posada D, Crandall KA (2000). TCS: a computer program to estimate gene genealogies. Mol Ecol 9: 1657-1659.

Crandall KA (1994). Intraspecific cladogram estimation: accuracy at higher levels of divergence. Syst Biol 43: 222-235. 
Farris JS, Källersjö M, Kluge AG, Bult C (1994). Testing significance of incongruence. Cladistics 10: 315-319.

Fu JZ, Murphy RW, Darevsky IS (2000). Divergence of the cytochrome $\mathrm{b}$ gene in the Lacerta raddei complex and its parthenogenetic daughter species: Evidence for recent multiple origins. Copeia 2: 432-440.

Guillou H, Carracedo JC, Torrado FP, Badiola ER (1996). K-Ar ages and magnetic stratigraphy of a hotspot-induced, fast grown oceanic island-El Hierro, Canary-Islands. J Volcanol Geothermal Res 73: 141-155.

Guillou H, Carracedo JC, Duncan RA (2001). K-Ar, 40Ar-39Ar ages and magnetostratigraphy of Brunhes and Matuyama lava sequences from La Palma Island. I Volcanol Geothermal Res 106: 175-194.

González P, Pinto F, Nogales M, Jiménez-Asensio J, Hernández M, Cabrera VM (1996). Phylogenetic relationships of the Canary Islands endemic lizard genus Gallotia (Sauria: Lacertidae), inferred from mitochondrial DNA sequences. Mol Phylogenet Evol 6: 63-71.

Ibáñez C, Fernández R (1985). Systematic status of the longeared bat Plecotus teneriffae Barret-Hamilton, 1907 (Chiroptera; Vespertilionidae). Säugetierkundliche Mitteilungen 32: 143-149.

Kocher TD, Thomas WK, Meyer A, Edwards SV, Pääbo S, Villablanca FX, Wilson AC (1989). Dynamics of mitochondrial DNA evolution in animals: amplification and sequencing of conserved regions. Proc Nat Acad Sci USA 86: 61966200 .

Juan C, Emerson B, Oromí P, Hewitt GM (2000). Colonization and diversification: towards a phylogeographic synthesis for the Canary Islands. Trends Ecol Evol 15: 104-109.

Mindell DP, Thacker CE (1996). Rates of molecular evolution: phylogenetic issues and applications. Ann Rev Ecol Syst 27: 279-303.

Norberg UM (1976). Aerodynamics, kinematics and energetics of horizontal flapping flight in the long-eared bat, Plecotus auritus. J Exp Biol 65: 179-212.

Pesole G, Gissi C, Chirico AD, Saccone C (1999). Nucleotide substitution rate of mammalian mitochondrial genomes. $J$ Mol Evol 48: 427-434.
Pestano J, Brown RP, Suarez NM, Fajardo S (2003). A mtDNA study of phylogeography and systematics of the Canary Island bats Pipistrellus and Hypsugo. Mol Phylogenet Evol., 26: 56-63.

Posada D, Crandall KA (1998) Modeltest: testing the model of DNA substitution. Bioinformatics 14: 817-818.

Pumo DE, Goldin EZ, Elliot B, Phillips CJ, Genoways $\mathrm{HH}$ (1988). Mitochondrial DNA polymorphism in three Antillean island populations of the fruit bat, Artibeus jamaicensis. Mol Biol Evol 5: 79-89.

Simon C, Franke A, Martin A (1990). The polymerase chain reaction: DNA extraction and amplification. In: Hewitt GM, Johnston AWB, Young JPW (eds) Molecular Techniques in Taxonomy, Springer: Berlin. NATO ASI series, series H: Cell Biology, Vol. 57, pp 329-357.

Swofford DL (1998). PAUP*, Phylogenetic Analysis Using Parsimony (*and other methods), Version 4. Sinauer Associates: Sunderland, MA.

Tamura K, Nei M (1993). Estimation of the number of nucleotide substitutions in the control region of mitochondrial-DNA in humans and chimpanzees. Mol Biol Evol 10: 512-526.

Templeton AR, Crandall KA, Sing CF (1992). A cladistic analysis of phenotypic associations with haplotypes inferred from restriction endonuclease mapping and DNA sequence data. III. Cladogram estimation. Genetics 132 619-633.

Thompson JD, Gibson TJ, Plewniak F, Jeanmougin F, Higgins DG (1997). The ClustalX windows interface: flexible strategies for multiple sequence alignment aided by quality analysis tools. Nucleic Acids Res 24: 4876-4882.

Trujillo, D (1991). Murcielagos de las Islas Canarias. Madrid: Minist. De Agri., Pesca y Alimentación. ICONA.

Van de Peer Y, De Rijk P, Wuyts J, Winkelmans T, De Wachter R (2000). The European Small Subunit Ribosomal RNA database. Nucleic Acids Res 28: 175-176.

Worthington-Wilmer J, Moritz C, Hall L, Toop J (1994). Extreme population structuring in the threatened Ghost Bat, Macroderma gigas: evidence from mitochondrial DNA. Proc $R$ Soc London B 257: 193-198. 